\title{
Carbapenem resistant Acinetobacter baumanii versus MRSA isolates in ICU in Clinical Center Skopje
}

\author{
Katja Popovska $^{1}$, Milka Zdravkovska ${ }^{2}$, Milena Petrovska ${ }^{1}$, Azis Pollozhani ${ }^{3}$ \\ ${ }^{1}$ Institute of Microbiology and parasitology, Faculty of Medicine, University “Ss Cyril and Methodius”, Skopje, Republic of Macedonia \\ ${ }^{2}$ Faculty of Medical Sciences, University “Goce Delchev”, Stip, Republic of Macedonia \\ ${ }^{3}$ Institute for Public Health of the Republic of Macedonia, Skopje, Republic of Macedonia
}

\section{Email address:}

katja.popovska@t-home.mk (K. Popovska)

\section{To cite this article:}

Katja Popovska, Milka Zdravkovska, Milena Petrovska, Azis Pollozhani. Carbapenem Resistant Acinetobacter Baumanii Versus MRSA Isolates in ICU in Clinical Center Skopje. European Journal of Preventive Medicine. Vol. 2, No. 2, 2014, pp. 20-24.

doi: $10.11648 /$ j.ejpm.20140202.11

\begin{abstract}
Backgorund: Acinetobacter baumannii is often referred to as the "Gram-negative methicillin-resistant Staphylococcus aureus", because it is frequently resistant to antibiotics. Clonal outbreaks of carbapenem-resistant and OXA-23-producing A. baumannii have been reported worldwide. Aim: The goal of this study was to promote the phenomenon of disbalance in endemic hospital ECO system which included increase of carbapenemase-resistant Acinetobacter baumanii on account of reduction of MRSA rate in surgical ICU and it's clonal relatedness as well as the specific precautions. Material nad Methods: Computer database from $1994-2012$ from surgical ICU patients in the Clinical Center Skopje was used as basic material for this study. Comparative study indicated 2007/8 as a break point period in which almost a twofold decrease of MRSA rates (from over $80 \%$ to $45 \%$ ) versus increased rates of Acinetobacter baumanii (from $29 \%$ to $40 \%$ ) was observed. In 2011 the very first eight strains of carbapenem resistant (resistant to imipenem and meropenem) A. baumannii, were observed. Disc diffusion and VITEK were used for antibiotic susceptibility testing. Resultes: Three distinct strains were detected by PFEGE and were designated as UKIM01AC-1 (5 strains), UKIM01AC-2 (two strains) and 642/2 (one strain). UKIM01AC-1 representatives were PCR positive for bla (OXA-23like), in addition to the bla (OXA- 51-like) gene which is intrinsic in Acinetobacter baumannii. All isolated strains belonged to European clone II lineage. Conclusion: This clone dispersed very fast in 2012 and achieved the rate of $61.9 \%$. This implicated changes in infection control precautions.
\end{abstract}

Keywords: Acinetobacter, Carbapenem Resistance, Clonal Distribution, Endemic ECO System

\section{Background}

Acinetobacter baumannii is often referred to as the "Gramnegative methicillin-resistant Staphylococcus aureus ", because it is frequently resistant to antibiotics. [1,2,3]

The epidemiologies of the two microbial species are very different and therefore, it is questionable whether the same control measures can be applied to the two pathogens. MRSA is primarily spread from person-to-person, whereas Acinetobacter infections often have an environmental source or reservoir. Allen and Green concluded that widespread aerial dissemination of Acinetobacter spp. occurred and this partly contributed to the environmental reservoir.

Referring to Allen and Green's study, Das et al. [4] hypothesised that heavily contaminated bed curtains, when moved would promote the airborne spread of Acinetobacter spp. Weernink et al. [5] investigated the airborne dispersal of Acinetobacter baumanii from patient's pillows. Using settle plates they found aerial dissemination from feather pillows, but not from synthetic pillows.

Numerous investigators have demonstrated the relative ease with which the clinical environment can become contaminated with pathogenic Acinetobacter species.

Dissemination by airborne or other routes can seed environmental reservoirs which either can start [6] or prolong outbreaks $[7,8]$. In institutions where outbreaks occur in ICUs, a common contaminated object in the environment can often be identified as the source. In contrast, in other hospitals, where epidemic infections have become endemic, the clinical and microbiological epidemiology of 
these infections often remains obscure. Allen and Green [4] were the first to suggest airborne dissemination of Acinetobacter-carrying particles. Investigating an outbreak of multiply-antibiotic resistant Acinetobacter anitratus in an ICU, a medical ward and three neurosurgical wards, they cultured the outbreak organism from 16 of 82 settle plates. Interestingly, all the positive plates were located within $3 \mathrm{~m}$ of the colonised patients.

There are studies showing the geographically widespread occurrence of multidrug-resistant A. baumannii strains, which suggested a clonal relatedness of these strains. [9, 10] Three international A. baumannii clones associated with multidrug resistance (European clones I, II, and III) have been reported).

Clonal outbreaks of carbapenem-resistant and OXA-23producing $A$. baumannii have been reported in many countries, such as Bulgaria, People's Republic of China, Brazil, Iraq, Afghanistan, Spain [9,10,11].

\section{Aim of the Study}

The goal of this study was to promote the phenomenon of disbalance in endemic hospital ECO system which included increase of carbapenemase-resistant Acinetobacter baumanii on account of reduction of MRSA rate in surgical ICU, by long-term monitoring, and the track whether disseminated strains of Acinetobacter were relevant. The final goal of this investigation was to promote specific precautions in order to minimise the acquisition and spread of these emerging infections that are difficult to treat.

\section{Methodology}

Comparative monitoring from database has indicated that there is a connection among emerging isolation of MDR Acinetobacter baumannii versus declination of MRSA isolates, both originating from surgical ICU patients in a long period of time.

$2007 / 8$ has been indicated as a break point period in which there was decriese of MRSA rates (from over $80 \%$ to $45 \%$ ) versus increased rates of Acinetobacter baumanii isolation (from $29 \%$ to $40 \%$ ) [Fig 1].

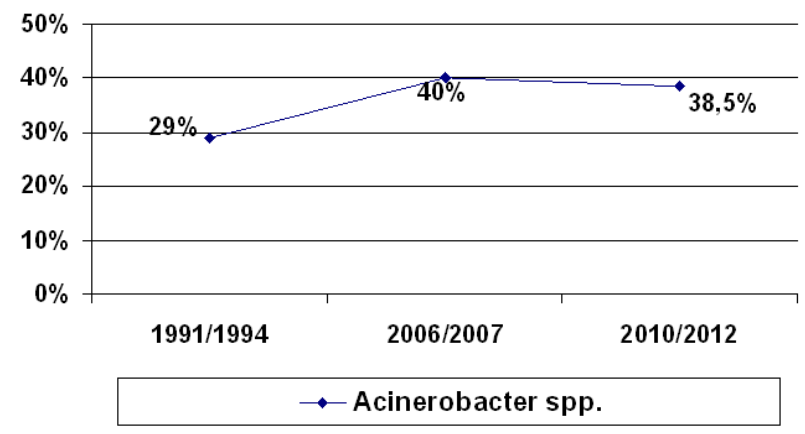

Figure 1. Acinetobacter isolation in ICU in the period of 1991/1994, 2006/2007 and 2010/2012 year
Table 1. Significance of isolation ratio for Acinetobacter isolates (1991/1994, 2006/2007 and 2010/2012 year)

\begin{tabular}{ll}
\hline Period & Student - t test of proportions \\
\hline $1991 / 1994-2006 / 2007$ & $\mathrm{p}=0,0020$ ( Sign.) \\
$1991 / 1994-2010 / 2012$ & $\mathrm{p}=0,0018$ (Sign.) \\
$2006 / 2007-2010 / 2012$ & $\mathrm{p}=0,7613$ (N.Sign.) \\
\hline
\end{tabular}

Acinetobacter baumanii is an important nosocomial pathogen in this Unit. In the period from 1991-1994 the rate of isolation was $29 \%(\mathrm{~N}=363)$. It significantly arised in the period from $2006-2007$ to $40 \%(\mathrm{~N}=355)$ and reflect this level in the next 5 years. On the other hand, MRSA became an important isolate in ICU in 1997 with over $80 \%$ rates sustaining percentage during the next decade, until 2007 when it was almost twice reduced. [Fig 2].

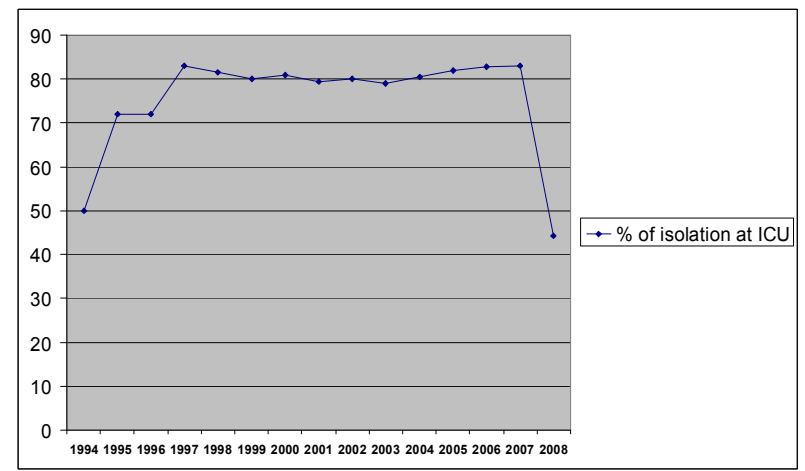

Fig 2. MRSA isolation in ICU in the period 1994-2008

Amikacin used to be a drug of choice in treatment of Acinetobacter baumanii infections during this long period of time, which has resulted in significant increasing $(p=0,0017)$ the resistance to this antibiotic in the period from 2005-2007 [Fig 3].

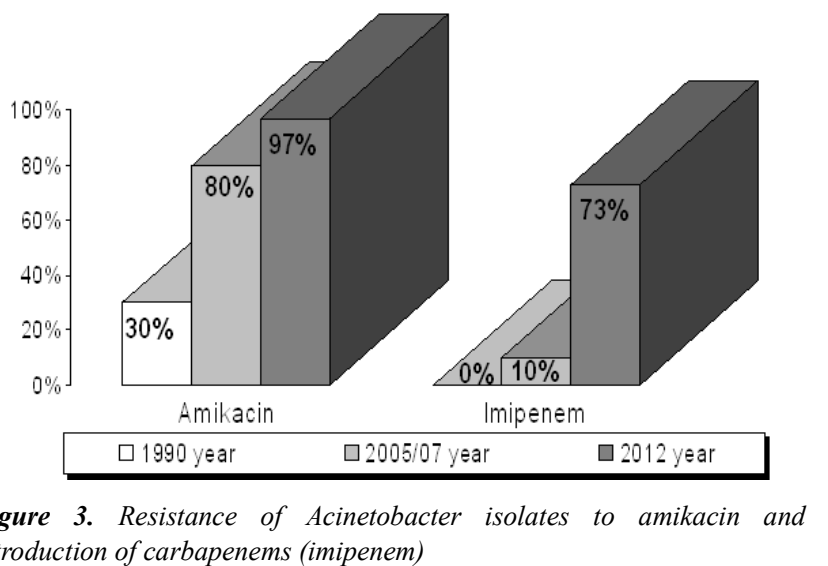

That is why carbapenems, firstly imipenem and later on meropenem, were involved in therapy in the early 2000 s.

\subsection{Design of the Study}

Antibiotic resistance among Acinetobacter baumanii has been observed in 3 periods of time: early period $1990-2004$, middle period 2005-2007 and recent period 2010-2012.

Strains of Acinetobacter baumanii originated from 
entubated patients in the surgical ICU. They were identified by classical microbiological procedure and confirmed by automatic VITEK technique). Susceptibility patterns to $\beta$ lactam antimicrobial drugs were determined by using a standard disk diffusion method according to published standards. Then strains of interest were determined by the agar dilution method, according to established NCCLS and confirmed by automatic VITEK test. Observation included 3 routinely used antibiotics, amikacin, imipenem and meropenem, since the observed strains were MDR.

Student's t-test of proportion was the epidemiological tool used in order to monitor the changes in resistance to carbapenems, from year to year, in the middle period and in the recent period of observation.

Eight strains of Acinetobacter baumanii were recognized to be resistant to both routinely used carbapenems (imipenem and meropenem) in the recent period, that was not the case with all other strains isolated ever before.

Clonal relatedness of these strains was assessed by pulsedfield gel electrophoresis (PFGE) and multilocus sequence typing in Health Protection Agency London by kindly collaboration with Prof Dr T.Pitt and Dr.Jane Turtone.[1,12]

Presence of the $b l a_{\mathrm{OXA}-23}$ gene was screened by PCR using specific primers (OXA-23-A 5'GGAATTCCATGAATAAATATTTTACTTGC-3' and OXA23-B 5'-CGGGATCCCGTTAAATAATATTCAGGTC-3'). Further PCR detection of these clones was performed in Institute of microbiology Skopje.

\section{Results}

\subsection{Acinetobacter Baumanii Ratio in Surgical ICU in the 21 Year Period}

The rates of Acinetobacter baumanii isolation in the 21 year period of observation in the surgical ICU, have showan a significant increas in the middle period (2006/7) with $40 \%(0,0018)$ rate of isolation. This trend of isolation has been observed in next the "recent period" $(38,5 \%)$, (2010-2012). [Fig 1].

The rate of isolation of MRSA as predominant pathogen in CARIC with $80 \%$ ratio has been found over ten year-period, until 2007/8, when it suddenly decreased to $40 \%$ [Fig 2].

\subsection{Antibiotic Susceptibility}

Comparison of antibiotic resistance to amikacin among the three investigated periods showed a significant ramp from $30 \%$ to $80 \%(p=0.00001)$ in the middle period $(2006 / 2007)$ and almost complete $(97 \%)$ resistance in the "recent period" (2010-2012) among 208 isolates of Acinetobacter. [Tab 1]

These data coincide with the introduction of imipenem in the treatment of surgical ICU patients, and registration the first imipenem-resistant strains (10\%) in the same period [Fig 3].

Ramps of resistance to imipenem from year to year, pointed a significant increase of imipenem-resisitance in 2007. [Fig 4].

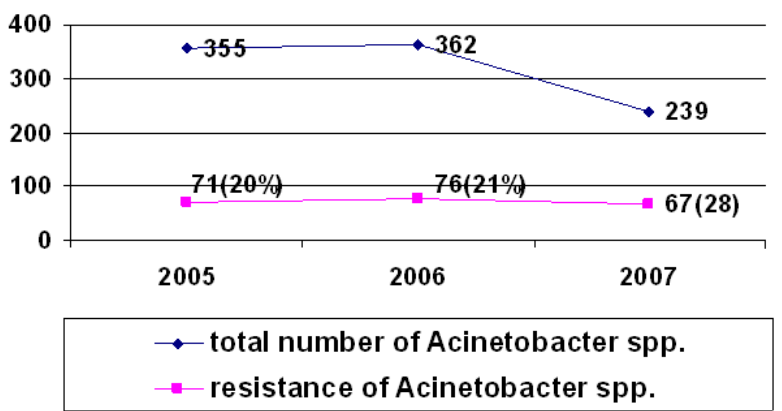

Figure 4. Dynamic of the resistance of Acinetobacter spp. to imipenem in the middle period - 2005/2007

Trend of spreading resistance to imipenem reached $73 \%$ $(p=0,00001)$ in the "recent period" $(2010-12)$ [Fig 5].

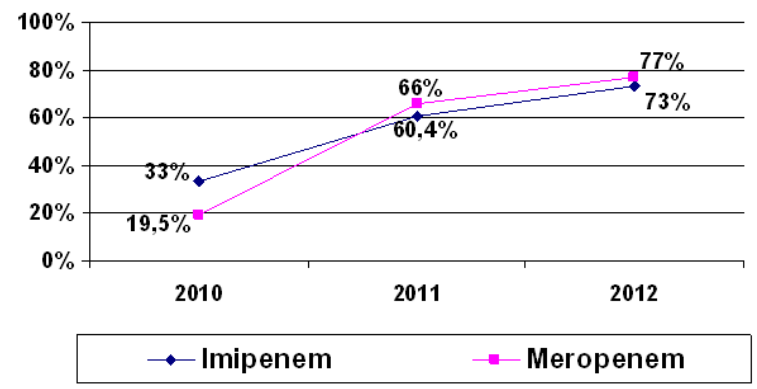

Fig 5. Imipenem and meropenem resistance of Acinetobacter baumanii in 2010/2012

According to Student"s t-test of proportion comparing 2011 and 2010, there was a significant $(\mathrm{p}=0,0003)$ elevation of resistance to imipenem, as well as to meropenem ( $\mathrm{p}=0,00001$ ) (from $33 \%$ to $60 \%$ to Imipenem, and from $19,5 \%$ to $66 \%$ for meropenem).

This trend was also observed in the first semester of 2012, but it was still not significant in 2011 for imipenem ( $\mathrm{p}-0,2010)$ as well as for meropenem ( $\mathrm{p}=0,2598)$ [Fig 5].

According to the data provided by the supplying service in the Surgery clinic, the use of relevant antibiotics in 2012 versus 2011 displayed $59 \%$ increase for meropenem (370amp. vs 180) and $40 \%$ decrease for imipenem (1010 amp. vs 2465 amp).

\subsection{Molecular Analysis of Resistant Strains}

Carbapenem-resistant Acinetobacter baumanii strains (resistant to both carbapenems) were isolated in 2011 by PFEGE analysis, and were similar in dendograms. Five strains had identical dendogram and were designated as UKIMAC1, 2 other similar strains designated as UKIMAC2 and only one unique strain [Tab 2].

Tab 2. PCR recognition of carbapenemase genes

\begin{tabular}{|c|c|c|c|c|}
\hline $\begin{array}{l}\text { Designition of } \\
\text { test strains }\end{array}$ & $\begin{array}{l}\text { Bla(OXA } \\
58 \text { like) }\end{array}$ & $\begin{array}{l}\text { Bla(OXA } \\
23 \text { like) }\end{array}$ & $\begin{array}{l}\text { Bla(OXA } \\
51 \text { like) }\end{array}$ & $\begin{array}{l}\text { Bla } \\
\text { (OXA } 40 \\
\text { like) }\end{array}$ \\
\hline $\begin{array}{l}\text { UKIM01 AC- } \\
1(\mathrm{~N}=5)\end{array}$ & negative & positive & positive & negative \\
\hline UKIM01AC-2 & negative & negative & positive & negative \\
\hline $642 / 2$ unique & negative & negative & positive & negative \\
\hline
\end{tabular}


PFGE analysis and random amplified polymorphic DNA (RAPD) generated by arbitrarily primed polymerase chain reaction of these eight isolates indicated 2 closely related genotypes ( 2 clones).

The OXA-23-producing isolates belonged to all genotypes. The presence of MDR OXA-23-producing $A$. baumannii in ICU has emphased the need to control the use of carbapenems.

\section{Discussion}

Continious monitoring is the basic tool in control of HAI, because it provides plenty of data about what happens in hospital ECO system, including appearance of MDR starins among endemic isolates and according to Rampell, changes in balance in hospital ECO system.

By long-term monitoring Acinetobacter baumanii has been confirmed as an important nosocomial pathogen in CARIC in the period 1991-1994, but it was increased in the period of 2007/8 - 2012, on account of MRSA. Similar findig for the same period of time, were refered from the investigation in some other countries such as Brazil or Taiwan $[17,18]$.

The comparative study indicated significant changes in EKO system in 2007/8 in investigated ICU. MRSA rates were almost double reduced and on the other side, rates of Acinetobacter baumanii significantly increased. [13]

Considering many differences in epidemiological characteristies of these two microorganisms, it was assumed that global or local conditions in the unit have been changed and/ it can be conetcet with the global changes in the climate [19,20]. Therefore Acinetobacter isolates became an important problem in this Unit, especially when carbapenem resistance was noticed in 2011 among eight strains which had been nonsusceptible to all other antibiotics tested by the agar dilution method.

All isolates of Acinetobacter were nonsusceptible to all of the antibiotics tested by the agar dilution method except amikacin until 2007 when resistance achieved 80\%. After that imipenem was involved as alteranative antibiotic therapy. In the next 5 years resistance among acinetobacters was spread very fast, almost completely to aminoglikozide (93\%) and very high to imipenem (73\%). Since therapeutic choice of Acinetobacter infections was small, meropenem was involved uncontrolled in therapeutic routine practice [Tab3].

Table 3. Use of carbapenems in ICU for period 2011-2012

\begin{tabular}{lll}
\hline & Ampulas used in 2011 & Ampulas used in 2012 \\
\hline meropenem & 180 & 370 \\
imipenem & 2465 & 1010 \\
gentamicin & 270 & 380 \\
\hline
\end{tabular}

This led to sudden development of meropenem-resistance. Among eight carbapenem resistant Acinetobacter baumanii tested representatives three distinct strains were detected by PFEGE. Five of them could possible belong to the same source - type UKIM01AC-1 and another two were connected - UKIM01AC 2. All representatives of UKIM01AC-1 were PCR positive for bla (OXA-23-like), in addition to the bla (OXA- 51-like) gene, which was intrinsic in Acinetobacter baumannii. All isolated strains belonged to European clone II lineage. [14], although there are data for A.baumanii isolates positive for bla (OXA-23-like) on american continent [17], This clone dispersed very fast in 2012 and achieved the rate of $61,9 \%(\mathrm{~N}=84)$.

\section{Conclusion}

Carbapenem resistant Acinetobacter baumanii (European) clone II, became significant pathogen in CARIC, replacing MRSA which was predominant cause of infections in over ten years period of time. This MDR clone expressed big capacity for intra/inter-hospital dissemination of the clone, which was confirmed by literature data and was due to unrational antibiotic usage in CARIC.

The new situation in this hospital ECO system indicated revision of hospital control strategies and applying new specific precautions. It was recommended: strong artificial control of air humidity, negative pressure in the critical room, proper care for bed-linen, replacement cotton with synthetic pillow, proper laundry of bed-linen, removal of mattress (once being Acineto-positive). Strong monitoring should be conduct over effect of recommended precautions for the following period of time.

\section{References}

[1] Silvia Munoz-Price and Robert A. Weinstein Acinetobacter Infection N Engl J Med 1271-1281.

(2008)

[2] Bou G, Cervero G, Angeles Dominguez M, Quereda C, Martinez-Beltran J.(2000) Characterization of a nosocomial outbreak caused by multiresistant Acinetobacterbaumannii strain with a carbapenem-hydrolyzing enzyme: high-level carbapenem resistance in $A$. baumannii is not due solely to the presence of $\beta$-lactamases. JClin Microbiol. 3299-3305.

[3] Ethan Rubinstein and Itxhak Levy (1999) Acinetobacter spp. and other non-fermentative pathogens causing nosocomial bacteremias Clin Microbiol Infect 2S29-2S32

[4] Allen KD, Green HT (1987) Hospital outbreak of multiresistant Acinetobacter anitratus: an airborne mode of spread? J Hosp Infect 110-119.

[5] Wernick, A (1995). Pillows, an unexpected source of acinetobacter. Journal of Hospital 189-199

[6] Raffaele Zarrilli, Margherita Crispino, Maria Bagattini, Elena Barretta, Anna Di Popolo Maria Triassi and Paolo Villari (2004) Molecular Epidemiology of Sequential Outbreaks of Acinetobacter baumannii in an Intensive Care Unit Shows the Emergence of Carbapenem Resistance; J. Clin. Microbiol 946-953

[7] Tankovic J, Legrand P, De Gaintes G, Cheminea V, Brun Buisson, Duval (1994) J.Characterization of a hospital outbreak of imipenem resistant Acinetobacter baumannii by phenotypic and genotypic typingmethods. J ClinMicrobiol 2677-2681. 
[8] Lolans K, Rice TW, Munoz-Price LS, Quinn JP (2006) Multicity outbreak of carbapenem-resistant Acinetobacter baumannii isolates producing the carbapenemase OXA-40. Antimicrob Agents Chemother 2941-2945

[9] Wen-Shyand Hsieh, Nai-Yu Wang, Jou-An Feng, Li Chuan Weng and Hsueh Hsia Wu (2012) Types and Prevalence of Carbapenem-Resistant Acinetobacter calcoacticusAcinetobacter baumanii complex in Northern Taiwan. J Clin Microbiol 2982-2986.

[10] Chaline S. Antonio, Patricia R. Neves, Micheli Medeiros and Elsa M. Mamikuza (2011) High prevalence of Carbapenem-Resistant Acinetobacter baumanii Carrying the blaOXA-143 Gene in Brasilian Hospitals. 1322-1233.

[11] Rodriguez-Bano J, Cisneros JM, Fernandez-Cuenca F (2004) Clinical features and epidemiology of Acinetobacter baumannii colonization and infection in Spanish hospitals. Infect Control Hosp Epidemiol.819-824

[12] Turton, J. F.(2006) The role of ISAba1 in expression of OXA carbapenemase genes in Acinetobacter baumannii. FEMS Microbiol. Lett. 258:72-77.

[13] Turton, J. F (2006) Identification of Acinetobacter baumannii by detection of the bla OXA-51-like carbapenemase gene intrinsic to this species. J. Clin. Microbiol 2974-2976.

[14] Evans B.A., Hamouda A., Amyes U.K (2013) The rise od carbapenem-resistant Acinetobacter baumanii. University of Manchester, Manchester, UK, 223-38.
[15] Paul G. Higgins1*, Cathrin Dammhayn1, Meredith Hackel2 and Harald Seifert1. (2009) Global spread of carbapenemresistant Acinetobacter baumannii. J Antimicrob Chemother 1091-1093

[16] Rea, E. Upshur, R. (2001). Semmelweis revisited: the ethics of infection prevention among health care workers. Canadian Medical Association Journal 1447-1448.

[17] Charline S. Antonio, Patricia R. Neves, Micheli Medeiros and Elsa M. Mamizuka (2010). High prevalence of carbapenem-resistant Acinetobacter baumanii carrying the bla OXA-143 gene in Brazillian Hospitals. Anim Agent Chemoter 1322-1323

[18] Wen-Sen-ShyangHsieh, Nai-Yu Wang, Jou-An Feng. Types and prevalence of carbapenem-resistant Acinetobacter calcoaceticus- acinetobacter baumaniii complex in Northern Taiwan (published before print 2013) Antimicrob Agent Chemoter doi.10.1128/AAC.0079-13

[19] Retailliau HF, Hightower AW, Dixon RE, Allen JR (1979) Acinetobacter calcoaceticus: a nosocomial pathogen with an unusual seasonal pattern. J Infect Dis 371-375.

[20] McDonald LC, Banerjee S, Jarvis WR (1999) NNIS System. Seasonal variation of Acinetobacter spp. Infections reported to the National Nosocomial Infections Surveillance (NNIS) System: 1987-1996 [abstract no 55]. Infect Control Hosp Epidemiol 1133-1137 